\title{
THICKNESS DETERMINATION OF SURFACE FILM FOR TEXTURED SPECIMENS BY X-RAY DIFFRACTION
}

\author{
LIU YU-SHU * and ZHAO YU \\ Public Laboratory of State Education Commission for High Temperature \\ Materials and Testing, Shanghai Jiao Tong \\ University, Shanghai 200030, China
}

(Received 20 March 1997)

A simple and convenient $\mathrm{X}$-ray diffraction method is proposed to determine the thickness of surface film for textured specimens. The analysis result for a synthetic specimen with surface film has proved that the method is applicable and reliable.

Keywords: Thickness determination; Surface film; Textured specimens; X-ray diffraction

\section{INTRODUCTION}

Improving and strengthening usabilities of materials by surface coating technique plays an important role in modern materials science and engineering. In order to control and estimate the production cost, one has to determine the thickness of the surface film. When the film or the matrix of the coated materials is crystalline and the film thickness is smaller than the depth penetrated by X-rays, the thickness can simply be determined by X-ray diffraction method, i.e., the absorption effect of incident and diffracted beams through the film (Friedman and Birk, 1946; Keating and Kammerer, 1958; Borie and Sparks, 1961). Usually the diffraction phase in these materials exhibits a preferred orientation of crystallites, namely texture, the diffracted intensity will depend on not only the film thickness itself, but also the texture. For this, some

\footnotetext{
* Corresponding author.
} 
effective methods to avoid the influence of texture on intensity were proposed and developed (Keating and Kammerer, 1958; Tao et al., 1984). The principle is not more than that one can eliminate the texture factor and finally obtain a unitary functional relation of diffracted intensity to film thickness by measurement of the intensity of a diffraction using two incident radiation with different wavelengths or measurement of the intensity from two orders of a diffraction. In order to further simplify the measurement procedure, a new method is introduced in the present work, by which neither the double-wavelength diffraction nor the double-order one, as has been stated above, is necessary. In the following we shall describe the fundamentals of the method in detail.

\section{FUNDAMENTALS OF THE METHOD}

\subsection{Experimental Arrangement}

The first measured position of a specimen is arranged after the backreflection technique (Schulz, 1949) with the specimen orientation $\{\alpha \beta\}$, as shown in Fig. 1(a). Here $\alpha$ is the rotation angle of the specimen about the intersection of the specimen surface and the diffraction plane, containing the incident and diffracted beam vectors, and $\beta$ is the rotation angle of the specimen about the normal to its surface. Then keeping $\alpha$ and $\beta$ fixed, the specimen will be rotated about the diffraction vector over $90^{\circ}$ as the second measured position (see Fig. 1(b)). Obviously, there will be the same texture factor and different paths of X-rays in the surface film for an analysed specimen in the two measured positions.

\subsection{Correction of Diffracted Intensity}

In back-reflection technique, using a flat specimen, a defocalization effect occurs due to an $\alpha$-tilt of the specimen. In the first case of measurement the defocalization may be negligible by the limit of a main slit in front of the specimen holder (Chernock and Beck, 1952). But in the second case a deviation of the irradiated specimen surface from the focussing sphere cannot be limited by the main slit (Liu, 1991). Hence, when the angle $\alpha$ is larger, one has to consider the effect of defocalization on measured intensities. In addition, the irradiated volume in the 


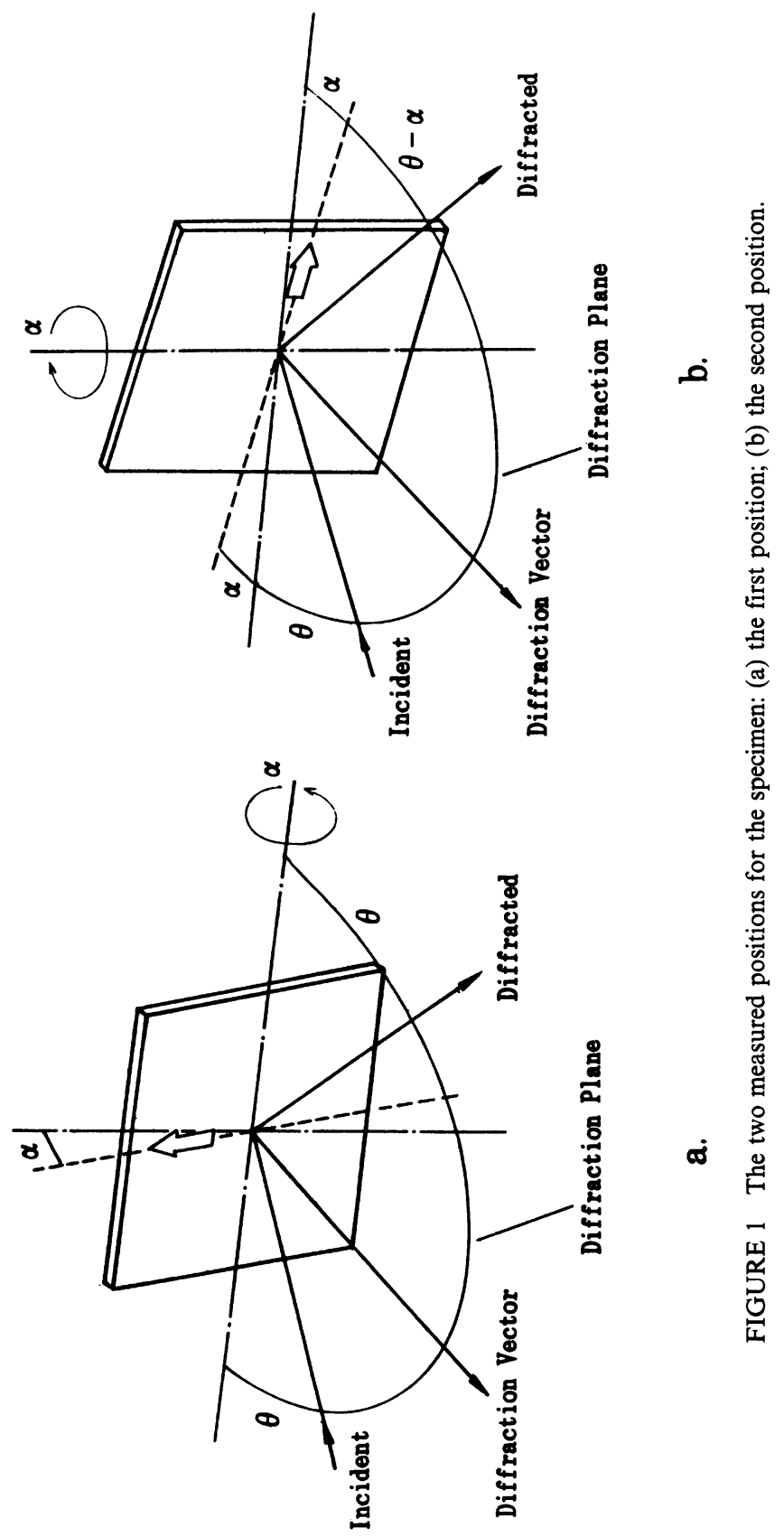


specimen will become different when there is a change from the first experimental position to the second one, that may also result in an evident influence on the measured result.

One can quantitatively consider the above-mentioned two effects on the intensity in the following way. Suppose $I_{\mathrm{c}}$ and $I_{\mathrm{c}}^{\prime}$ are the diffracted intensities from a correcting specimen in the absence of film, separately in the first and second cases (with certain $\alpha$ and $\theta$ ), then we take

$$
C=I_{\mathrm{c}}^{\prime} / I_{\mathrm{c}}
$$

as the correction factor of the intensity in the second experimental case, with respect to the first case, for the corresponding $\alpha$ and $\theta$.

\subsection{Film Diffraction}

Figure 2(a) shows the diffraction geometry in the section of a specimen with surface film along the diffraction plane, corresponding to the first measured position. Suppose the thickness of the film is $D$, the visible film thickness in the section should be $D / \cos \alpha$. Consider the diffraction at a particular lattice plane $(h k l)$ in a thin layer of the film with the thickness $\mathrm{d} x$, located at a depth $x$ below the surface (as indicated by shading in Fig. 2(a)), the total length of the path passed by the incident and diffracted beams with Bragg-angle $\theta$ in the film is $2 x / \sin \theta$. Given that the irradiated area on the specimen surface for $\alpha=0^{\circ}$ is $A_{0}$, and the film has an absorption coefficient $\mu_{\mathrm{f}}$, the diffracted intensity from the thin layer may be described in the form

$$
\mathrm{d} I=I_{0} V_{\mathrm{d}} R_{h k l} P_{h k l}(\alpha \beta) \exp \left(-2 \mu_{\mathrm{f}} x / \sin \theta\right) A_{0} \mathrm{~d} x,
$$

where $I_{0}$ is the incident intensity, $V_{\mathrm{d}}$ the volume fraction of the diffraction phase in the film, $R_{h k l}$ the reflectivity of the lattice plane $(h k l)$, $P_{h k l}(\alpha \beta)$ the $(h k l)$-pole density in the specimen direction $\{\alpha \beta\}$, $\exp \left(-2 \mu_{\mathrm{f}} x / \sin \theta\right)$ the absorption factor for film diffraction, $A_{0} \mathrm{~d} x$ the volume of the considered thin layer in the film. After Schulz's consideration, the elementary volume is independent of $\alpha$ in this case (Schulz, 1949). The total intensity, diffracted by all the irradiated film, can be obtained by integrating the above intensity $\mathrm{d} I$ over $x$ from 0 to 


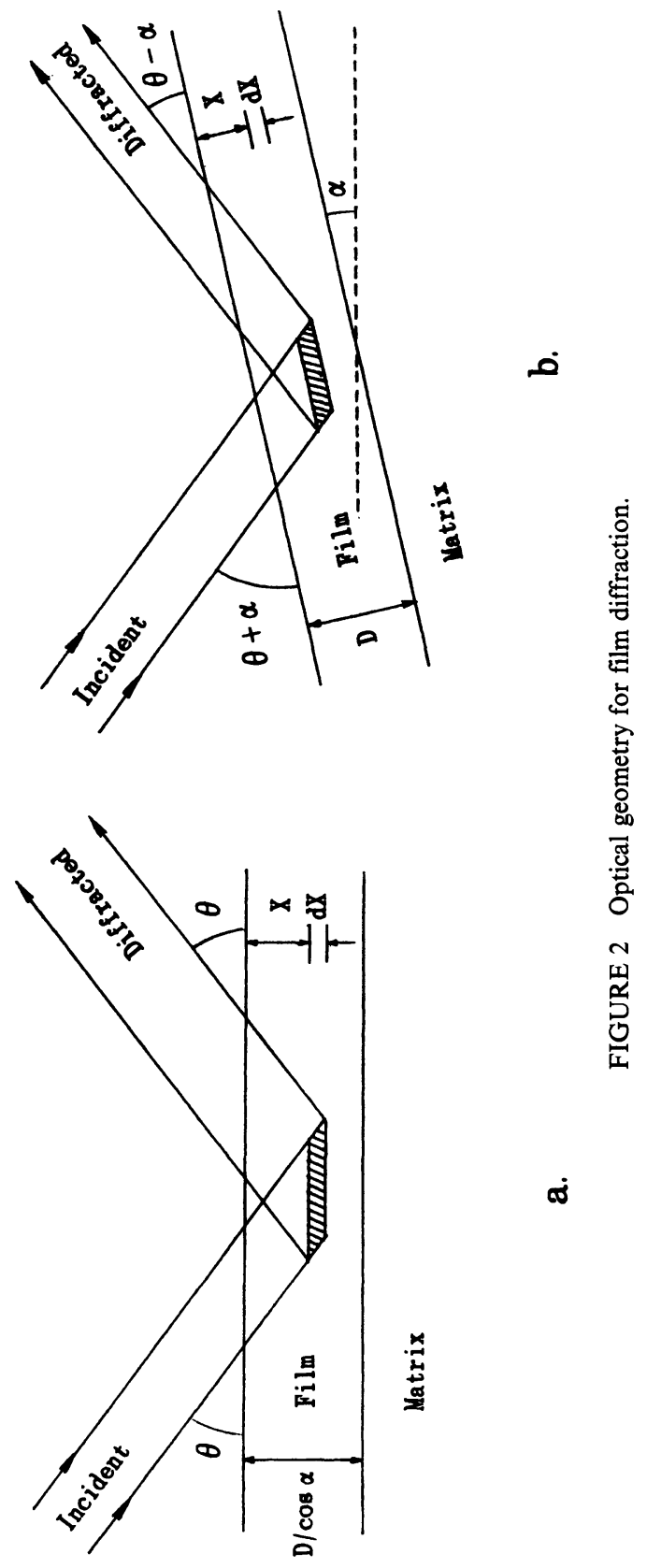


$D / \cos \alpha$, i.e., over the depth penetrated by $\mathrm{X}$-rays through the film:

$$
\begin{aligned}
I= & I_{0} V_{\mathrm{d}} R_{h k l} P_{h k l}(\alpha \beta) A_{0} \sin \theta /\left(2 \mu_{\mathrm{f}}\right) \\
& \times\left\{1-\exp \left[-2 \mu_{\mathrm{f}} D /(\cos \alpha \sin \theta)\right]\right\} .
\end{aligned}
$$

For the film diffraction in the second measured position (see Fig. 2(b)), the path lengths of the incident and diffracted beams separately become $x / \sin (\theta+\alpha)$ and $x / \sin (\theta-\alpha)$. The intensity of the $(h k l)$-diffraction from the thin layer, as shown by shading in Fig. 2(b), is

$$
\begin{aligned}
\mathrm{d} I^{\prime}= & I_{0} V_{\mathrm{d}} R_{h k l} P_{h k l}(\alpha \beta) \exp \left\{-\mu_{\mathrm{f}} x[1 / \sin (\theta+\alpha)\right. \\
& +1 / \sin (\theta-\alpha)]\} C A_{0} \mathrm{~d} x
\end{aligned}
$$

where $\exp \left\{-\mu_{\mathrm{f}} x[1 / \sin (\theta+\alpha)+1 / \sin (\theta-\alpha)]\right\}$ is the absorption factor of the film in the new measured position. Due to a change of the irradiated volume and a defocussing effect, the correction factor $C$ should here be introduced in Eq. (4). The total intensity of film diffraction in this case will be equal to the integration of $\mathrm{d} I^{\prime}$ over $x$ from 0 to $D$ :

$$
\begin{aligned}
I^{\prime}= & I_{0} V_{\mathrm{d}} R_{h k l} P_{h k l}(\alpha \beta) C A_{0}[\cos (2 \alpha)-\cos (2 \theta)] /\left(4 \mu_{\mathrm{f}} \sin \theta \cos \alpha\right) \\
& \times\left\{1-\exp \left[4 \mu_{\mathrm{f}} D \sin \theta \cos \alpha /(\cos 2 \theta-\cos 2 \alpha)\right]\right\}
\end{aligned}
$$

Substituting the experimentally measured intensities, $I$ and $I^{\prime}$, into Eqs. (3) and (5), one can determine the film thickness $D$.

\subsection{Matrix Diffraction}

The optical geometries of matrix diffraction with respect to the two experimental arrangements are seen in Fig. 3(a) and (b). Similar to the discussion about film diffraction, we take the $\left(h^{\prime} k^{\prime} l^{\prime}\right)$-diffraction from the thin layer in the matrix, as shown in Fig. 3, into account, then the absorption factors of the specimen corresponding to the two measured positions are, separately, $\exp \left\{-2 / \sin \theta^{\prime}\left[\mu_{\mathrm{f}} D / \cos \alpha+\mu_{\mathrm{m}} x\right]\right\}$ and $\exp \left\{-\left(\mu_{\mathrm{f}} D+\mu_{\mathrm{m}} x\right)\left[1 / \sin \left(\theta^{\prime}+\alpha\right)+1 / \sin \left(\theta^{\prime}-\alpha\right)\right]\right\}$, where $\mu_{\mathrm{m}}$ is the absorption coefficient of the matrix. By integrating the diffracted intensity of the considered matrix element over the whole irradiated volume in the matrix (in other words, over $x$ from 0 to $\infty$ ), the total 


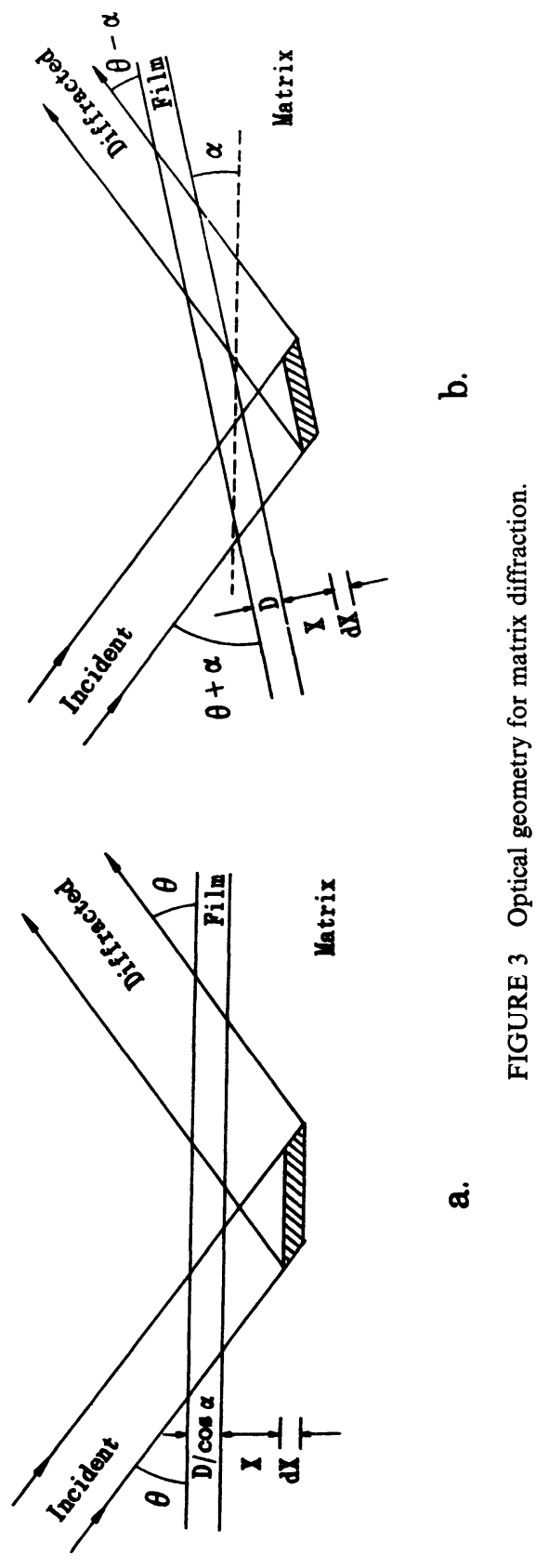


intensities of matrix diffraction in the two measuring cases are expressed separately as

$$
\begin{aligned}
I= & I_{0} V_{\mathrm{d}}^{\prime} R_{h^{\prime} k^{\prime} l^{\prime}} P_{h^{\prime} k^{\prime} l^{\prime}}(\alpha \beta) A_{0} \sin \theta^{\prime} /\left(2 \mu_{\mathrm{m}}\right) \\
& \times \exp \left[-2 \mu_{\mathrm{f}} D /\left(\cos \alpha \sin \theta^{\prime}\right)\right]
\end{aligned}
$$

and

$$
\begin{aligned}
I^{\prime}= & I_{0} V_{\mathrm{d}}^{\prime} R_{h^{\prime} k^{\prime} l^{\prime}} P_{h^{\prime} k^{\prime} l^{\prime}}(\alpha \beta) C A_{0}\left[\cos (2 \alpha)-\cos \left(2 \theta^{\prime}\right)\right] / \\
& \left(4 \mu_{\mathrm{m}} \sin \theta^{\prime} \cos \alpha\right) \exp \left[4 \mu_{\mathrm{f}} D \sin \theta^{\prime} \cos \alpha /\left(\cos 2 \theta^{\prime}-\cos 2 \alpha\right)\right]
\end{aligned}
$$

The film thickness $D$ may also be determined from the two experimentally measured intensitites $I$ and $I^{\prime}$ by the simultaneous Eqs. (6) and (7).

\section{EXPERIMENT AND RESULT}

In order to check the introduced method, a synthetic specimen with surface film was prepared by combining a rolled nickel film and a rolled copper sheet with strong texture, which are on hand, with each other to simulate coated materials. The thickness of the nickel film is $5.521 \mu \mathrm{m}$, determined from its weight, density and surface sizes.

The experiment was carried out with $\mathrm{Cu} \mathrm{K} \alpha$-radiation in the diffractometer D/MAX-3A. For measurement the tilting angle $\alpha$ of the specimen was $5^{\circ}$, and a $\beta$-rotation with higher speed was executed to increase the experimental statistical accuracy. The texture factor, thus, is independent of $\beta$. The diffracted intensity from the lattice plane (111) of the 'matrix' $\mathrm{Cu}\left(\theta=21.38^{\circ}\right)$ was measured. This peak is well resolved from neighbouring peaks. Due to a broadening of the diffraction peak caused by various reasons, a step-scanning method was applied to obtain the complete peak profile. Then the $\mathrm{K} \alpha_{1}$-peak was separated from $\mathrm{K} \alpha_{2}$, and the integral intensity of the former was substituted for $I$ in Eq. (6) or $I^{\prime}$ in Eq. (7), depending on the corresponding measured position. The correction factor $C$ was obtained by the intensities of the same diffraction from a copper sheet without film in the two measured positions. The thickness of the nickel film was finally determined by Eqs. (6) and (7). The experimental and calculated results are shown in Table I: 
Table I Some experimental and calculated results

\begin{tabular}{lccc}
\hline $\begin{array}{l}\text { Diffracted Intensity } \\
(\mathrm{pcs})\end{array}$ & Correction Factor $C$ & $\begin{array}{c}\text { Ni-Film Thickness } \\
(\mu \mathrm{m})\end{array}$ \\
\hline$I$ & 4258 & 0.8613 & 5.7468 \\
\hline$I^{\prime}$ & 2279 & & \\
\hline
\end{tabular}

Taking the possible errors, such as the inhomogeneity of the film thickness or the roughness of the specimen surface, into account, we may think the calculated result is satisfactory, as compared with the above-mentioned weight method.

\section{CONCLUSIONS}

The thickness of a surface film on textured materials can easily be determined by the X-ray diffraction method, recommended in the paper, when the film thickness is smaller than the penetrated depth of $\mathrm{X}$-rays.

The advantage of the method over others is to need neither the double-wavelength nor the double-order diffraction, that may further simplify the experimental procedure.

The analysis for a synthetic specimen with surface film indicates that the result is accurate enough and the method is applicable.

\section{References}

Borie, B. and Sparks, C.J. (1961), Acta Crystal., 14, 569.

Chernock, W.P. and Beck, P.A (1952), J. Appl. Phys., 23, 341.

Friedman, H. and Birk, L.S. (1946), Rev. Sci. Instrum., 17, 99.

Keating, D.T. and Kammerer, O.F. (1958), Rev. Sci. Instrum., $29,34$.

Liu, Y.S. (1991) Thesis, Clausthal, 36.

Schulz, L.G. (1949), J. Appl. Phys., 20, 1030.

Tao, K. et al. (1984), Physical Testing and Chemical Analysis, Part A, 20(1), 19 (in Chinese). 